ISSN: 0514-7336

DOI: http://dx.doi.org/10.14201/zephyrus201372175182

\title{
FÍBULA ROMANA “DE RODILLA” (KNIEFIBELN) PROCEDENTE DE HIJES (GUADALAJARA)
}

\section{Kniefibeln from Hijes (Guadalajara, Spain)}

\author{
María Luisa CERDEÑo* y Emilio GAMO* \\ *Dpto. de Prehistoria. Facultad de Geografía e Historia. Avda. Profesor Aranguren, s/n. 28040 Madrid. Correo-e: \\ mluisac@ucm.es; emiliogamo@hotmail.com
}

Recepción: 28/11/2012; Revisión: 20/05/2013; Aceptación: 18/06/2013

BIBLID [0514-7336 (2013) LXXII, julio-diciembre; 175-182]

RESUMEN: En este artículo se quiere llamar la atención sobre la aparición de una "fíbula de rodilla" (Kniefibeln) en el yacimiento romano de Los Arroyos II en Hijes (Guadalajara); su presencia es muy significativa dado que se trata de un tipo propio de Europa central y este es el primer ejemplar conocido en la Península Ibérica. El estudio tipológico de los elementos materiales es imprescindible en la investigación arqueológica pero, más allá de su mera clasificación, deben ser considerados como parte de las prácticas sociales y como reflejo de las personas que los fabricaron y utilizaron. La presencia de esta fíbula en la Meseta, lugar muy alejado de los territorios centroeuropeos, puede indicar movimientos de personas, presumiblemente militares termestinos, entre los ejércitos que defendían el limes germánico y renano-danubiano. Esta conclusión está avalada no solo por los datos que aporta la arqueología, sino también por documentos epigráficos, lo que confirma la necesidad metodológica de manejar diferentes fuentes de información para abordar un mismo estudio histórico.

Palabras clave: Fíbula de rodilla. Territorium de Tiermes. Ejército romano. Limes germánico y renanodanubiano. Datos arqueológicos y epigráficos.

ABSTRACT: In this paper we want to highlight on the recent finding of one Kniefibeln in the roman site of "Los Arroyos II", Hijes (Guadalajara, Spain). Its presence is really significant because this kind of fibeln basically came from Central Europe and this is the first one find in the Iberian Peninsula. The typological study is an essential part of the archaeological research but, on top of the basic technical aspects, it must be analized its social uses as well as the people who made and dressed it. The finding of this fibeln in Spanish Plateau, far from the place it was manufactured, indicates population movement, supposedly soldiers from Tiermes, moved to defend the Germanic and Rhenish-Danubian limes. This conclusion is supported not only by archaeological data but also by epigraphic documents. This tell us that, under a methodological point of view, we have to focus the historical studies from different information sources.

Key words: Kniefibeln. Territorium of Tiermes. Roman army. Germanic and Rhenish-Danubian limes. Archaeological and epigraphic data. 


\section{Introducción}

La atención pormenorizada hacia determinados objetos materiales de las antiguas culturas ha sido considerada en algunos momentos de la investigación como una forma tradicional del trabajo arqueológico, puesto que puede derivar en un excesivo empirismo que impida sobrepasar el escalón meramente descriptivo. Pero para llegar al análisis, la interpretación y, en definitiva, a la formulación de hipótesis no se puede carecer de un sólido registro que la sustente, cuya formación pasa por la identificación, descripción y clasificación de los datos disponibles. Desde hace ya tiempo se ha vuelto a reivindicar el papel que debe ocupar la cultura material, que no es solo un conjunto de elementos estáticos si aceptamos que las prácticas socioeconómicas involucran y dimensionan el mundo de los objetos (Castro et al., 1996: 252), por lo que a través de ellos llegaremos a saber quiénes y cómo fueron sus fabricantes y destinatarios.

Por ello hemos centrado nuestra atención en esta fíbula ya que se trata de un tipo desconocido hasta ahora en la Península que, sin embargo, está muy identificado en otros ámbitos geográficos bien distantes. Las fíbulas se convirtieron en un objeto ampliamente utilizado, sin duda porque solucionaban con eficacia el problema de sujetar la vestimenta y aquella extensa difusión y uso les concede un importante valor cronotipológico ya que los modelos se sucedieron y variaron continuamente en el tiempo y el espacio. Más allá de su indudable funcionalidad, tuvieron en muchas ocasiones un valor simbólico y sobre todo social que ayuda a identificar a sus usuarios. La que ahora estudiamos es un modelo perfectamente registrado en contextos del s. II d. C. y creemos que es posible establecer una justificación histórica clara que explique la lejanía de este hallazgo respecto a su núcleo de dispersión mayoritario.

Esta fíbula de rodilla se conserva en la exposición permanente del Museo de San Gil de Atienza ${ }^{1}$ donde fue depositada en el año 1980, tras su aparición casual durante la realización de labores agrícolas en la comarca. Concretamente procedía del paraje denominado "Los Arroyos", lugar donde

${ }^{1}$ N. ${ }^{o}$ de inventario: 0120/ATSG 0169. Agradecemos a D. Agustín González, párroco de Atienza y responsable del Museo, las facilidades para acceder a la pieza. se han realizado diferentes hallazgos arqueológicos desde hace más de un siglo. El término municipal de Hijes es más conocido porque a mediados del s. XIX se encontró una necrópolis celtibérica en la que se realizaron cuatro campañas durante los años $1840,1845,1850$ y 1912, esta última dirigida por el marqués de Cerralbo, que la incluyó en la lista de sus famosos cementerios ${ }^{2}$ (Aguilera y Gamboa, 1911: 14; 1916: 31, 58), suficientemente estudiados desde entonces.

Por el contrario, el hábitat romano ahora objeto de nuestro interés quedó prácticamente en el olvido, a pesar de estar situado a $17 \mathrm{~km}$ de la ciudad de Tiermes, a cuyo territorio se adscribió, sin duda, en época imperial. Se conocía su existencia por la correspondencia -conservada en la Real Academia de Bellas Artes de San Fernando- que mantuvo la Comisión Central de Monumentos con su Sección en Guadalajara, en la que aparece mencionado el hallazgo de una villa romana durante las excavaciones realizadas por el párroco de la localidad en el año 1845 en un lugar poco preciso (Gómez-Pantoja y López, 2004: 153-160) y al que la moderna investigación no había vuelto a prestar atención.

Como parte de la actividad arqueológica que venimos desarrollando desde hace años en la provincia, enfocada principalmente al estudio de los enclaves celtibéricos y romanos, llevamos a cabo prospecciones para localizar algunos yacimientos que se conocían solo por referencias, contextualizarlos adecuadamente y así conseguir una documentación sistemática que pudiera unirse a la obtenida de los materiales conservados en diferentes museos, procedentes de aquellas excavaciones antiguas y de hallazgos casuales. Por ello, en el año 2010 realizamos una prospección sistemática en la vega de Hijes durante la que se localizó la ubicación de la necrópolis celtibérica ("Los Arroyos I") y del yacimiento romano ("Los Arroyos II") $)^{3}$ ya

\footnotetext{
${ }^{2}$ Además de la obra publicada en 1916 de E. Aguilera y Gamboa hemos consultado su texto inédito de 1911: Páginas de la historia patria por mis excavaciones arqueológicas, t. IV , p. 14.

${ }^{3}$ La prospección fue realizada por E. Gamo en el marco del proyecto "La época romana republicana en la actual provincia de Guadalajara, siglos II-I a. C.”, concedido dentro del Programa de Subvenciones para financiar investigaciones sobre el Patrimonio Histórico, Artístico y Cultural de Castilla-La Mancha (DOCM n. ${ }^{\circ}$ 54, de 18/03/2010).
} 
que están prácticamente juntos. Se sitúan en una suave ladera en la margen izquierda del arroyo de Pajares, sobre una parcela dedicada al cultivo de secano en la que se conservan en superficie materiales romanos extendidos a lo largo de casi 3 ha.

Todo parece indicar que se trata de la pars rustica de una villa cercana, es decir, un lugar de marcado carácter funcional vinculado a la explotación agropecuaria a juzgar por las numerosas ruedas de molino giratorias de grandes dimensiones (Fig. 1a) y la ausencia de elementos constructivos característicos de la pars urbana de las villae, como son los mosaicos o las columnas. También aparecieron en superficie fragmentos muy rodados de TSG de forma indeterminada, TSH (formas Drag. 15/17, 27 y 37), TSHT (forma Drag. 37 tardía), fragmentos de cerámica común romana (forma Vegas 1) y fragmentos de cerámica pintada de tradición indígena de difícil identificación.

Además, debieron existir restos constructivos puesto que en la mencionada correspondencia decimonónica se dice que se había localizado una "considerable exten-
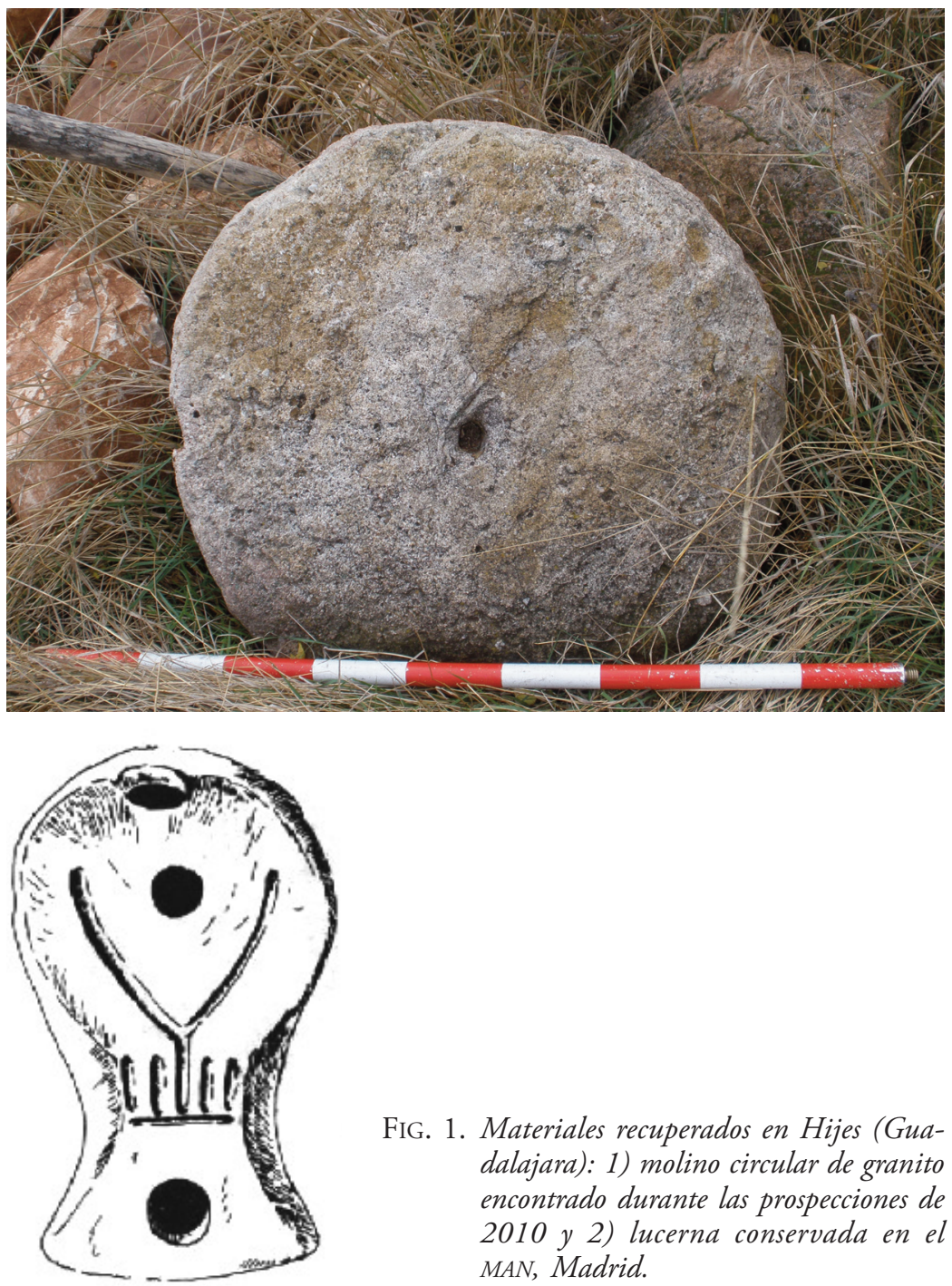

FIG. 1. Materiales recuperados en Hijes (Guadalajara): 1) molino circular de granito encontrado durante las prospecciones de 2010 y 2) lucerna conservada en el MAN, Madrid.

sión de cimientos de bastante

buena construcción" o muros "de piedra formados con cal y canto de bastante extensión y a todo coste" (Gómez Pantoja y López, 2004: 157-158). Ello parece corroborado por el hallazgo en superficie, durante nuestras prospecciones, de numerosas tégulas e ímbrices, así como de sillares desplazados y revueltos por la acción de los tractores.

Todos estos materiales localizados en Hijes hay que relacionarlos con las otras piezas conocidas de antiguo y conservadas en diferentes museos. Aparte de la fíbula de rodilla, en el Museo de San Gil de Atienza se expone un pondus cerámico de sección rectangular. El Museo Arqueológico Nacional conserva una lucerna itálica de la forma Dressel 22 (Fig. 1.2) en forma de lira de cuerpo aplanado y ansa transversal con infundibulum, decorada con incisiones (Amaré, 1987: 11, 28) y en el Museo de Molina de Aragón están depositadas dos pesas metálicas, una de bronce con decoración estriada y otra de hierro de forma troncocónica ${ }^{4}$. Se sabe que también había monedas porque las cartas de la Comisión indican que durante la campaña de 1845 se descubrieron tres: "dos del Emperador Graciano y la otra de Constantino Pío Félix Augusto" (GómezPantoja y López, 2004: 157).

\footnotetext{
${ }^{4} \mathrm{~N} .{ }^{\text {os }}$ de inventario: MOL/0224 y MOL/0225.
} 
El reconocimiento directo del lugar y los hallazgos realizados, unidos a la revisión de todos los materiales anteriores, permiten confirmar que se trataba de un hábitat rural romano con una ocupación prolongada en el tiempo, que se extiende desde la primera mitad del s. I a. C. hasta época bajoimperial en los ss. IV-V d. C. Este contexto arqueológico encaja adecuadamente con la fíbula que presentamos y permite aceptar la información de su hallazgo en este lugar.

\section{Estudio de la fíbula}

\subsection{Adscripción tipológica}

La fíbula estudiada (Fig. 2) corresponde al tipo Kniefibeln, que podemos traducir literalmente por "fíbula de rodilla", definido así por Kovig (1937). Concretamente se asimila a la variante 7.4 subtipo de cabecera semicircular de Kropf y Nowak (2000: 89), equivalente al tipo 13D de Jobst (1975) que fue quien realizó una amplia sistematización del modelo a partir de las piezas halladas en el campamento romano de Lauriacum, en la provincia de Noricum.

Es de bronce, de pequeño tamaño y está formada por varias piezas independientes: la aguja se enrolla en su extremo proximal sobre un corto eje, formando cuatro espiras a cada lado, unidas por cuerda exterior; el puente es una pieza cuya cabecera tiene forma semicircular que descansa sobre el muelle y está decorada con 4 filas de pequeños triángulos o dientes de lobo troquelados, siguiendo los bordes; bajo esta cabecera se funde una chapa perpendicular que la une al centro del eje; el puente o arco forma un ángulo muy pronunciado, que da nombre al tipo general y termina en un chato y corto pie ligeramente apuntado hacia arriba; por debajo del ángulo que marca el pie, se adosa perpendicularmente una lámina rectangular, doblada en su extremo para formar la mortaja o portaaguja. Se conserva completa y sus dimensiones son $36 \mathrm{~mm}$ de longitud y $24 \mathrm{~mm}$ de altura.

Las fíbulas tipo rodilla -Kniefibeln- concentran su área de dispersión en los territorios de Europa central (Figs. 3-4), especialmente en las antiguas regiones de Dacia, Pannonia, Noricum, Germania y Britannia, conociéndose pocos ejemplares en el resto de Europa y ninguno en Hispania, según hemos comprobado en trabajos especializados (Erice, 1995; Mariné, 2001, 2007; Ponte, 2007).

Se han fechado genéricamente entre mediados del s. II y el s. III d. C. (Kropf y Nowak, 19981999: 30), aunque el momento de inicio del modelo de cabecera semicircular ha sido objeto de debate. Jobst (1975: 66) defendió la difusión de este subtipo en la segunda mitad del s. II, mientras otros investigadores han propuesto una cronología algo más temprana, como Berecz (1991: 165), que sostuvo el inicio de su fabricación a comienzos de dicha centuria y su perduración hasta principios del s. III. Otros autores han defendido también una cronología temprana, proponiendo que su aparición se produjo en época de Adriano y que su uso perduró solamente hasta finales del s. II (Riha, 1979: 84, 85, 159).
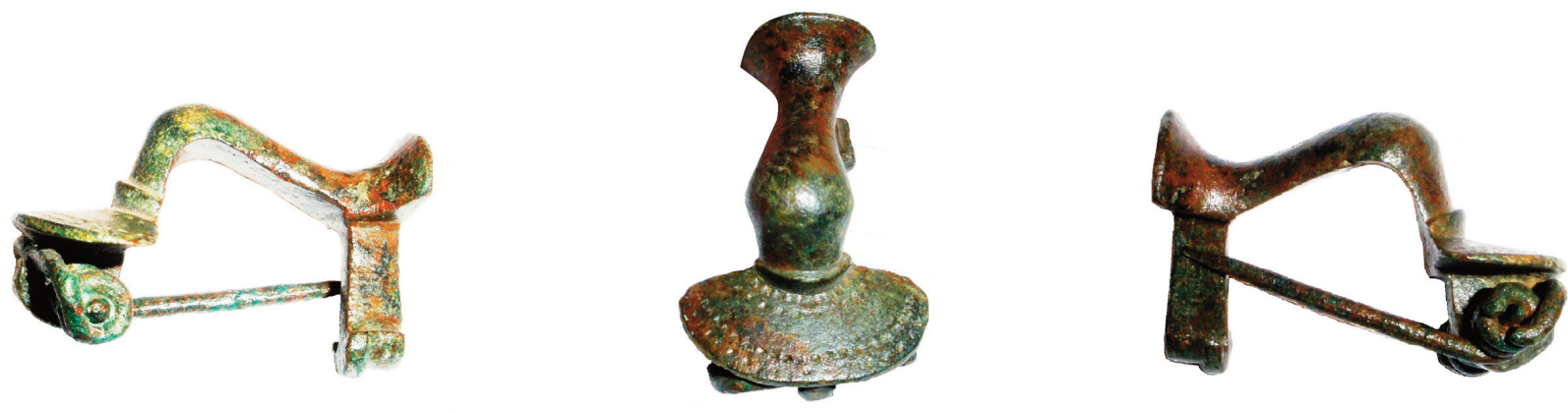

FIG. 2. Fíbula de rodilla con cabecera semicircular procedente de Hijes. 
Bogdan y Cocis (2006: 221) han estudiado los hallazgos en Dacia, donde tuvieron gran difusión, y han concluido que las primeras piezas de tipo Kniefibeln corresponden al reinado de Trajano -post 106- y perduraron hasta el último tercio del s. II d. C.; su principal argumento es que sólo una de las 100 fíbulas documentadas en el campamento legionario de Potaissa (Rumania) era de este tipo y dicho campamento fue construido entre 168170 d. C. Probablemente el modelo comenzó a fabricarse en Noricum y desde allí se extendería a las provincias germanas y danubianas, llegando hasta Britannia e incluso fuera de los límites del imperio (Jobst, 1975: 65; Patek, 1942: 59-62; Hattatt, 1985: 119223; Vaday, 1989: 305).

La amplia difusión de estas piezas (Fig. 4) se ha justificado porque se las considera propias de la indumentaria de los soldados y su circulación se produciría al ritmo al que se movían las tropas. Sin embargo, algunos autores discrepan de esta afirmación argumentando que, en ocasiones, los hallazgos se han producido en enclaves de carácter puramente civil, como es el caso de Flavia Solva y que además aparecen en algunos territorios del interior del Limes renano-danubiano (Kropf y Nowak, 1998-1999: 30).

\subsection{Otros elementos relevantes}

El hallazgo de una fíbula de este tipo en una zona tan distante como la Meseta inclina a pensar que se debió a la presencia de soldados hispanos entre las tropas allí destacadas que, de regreso a su
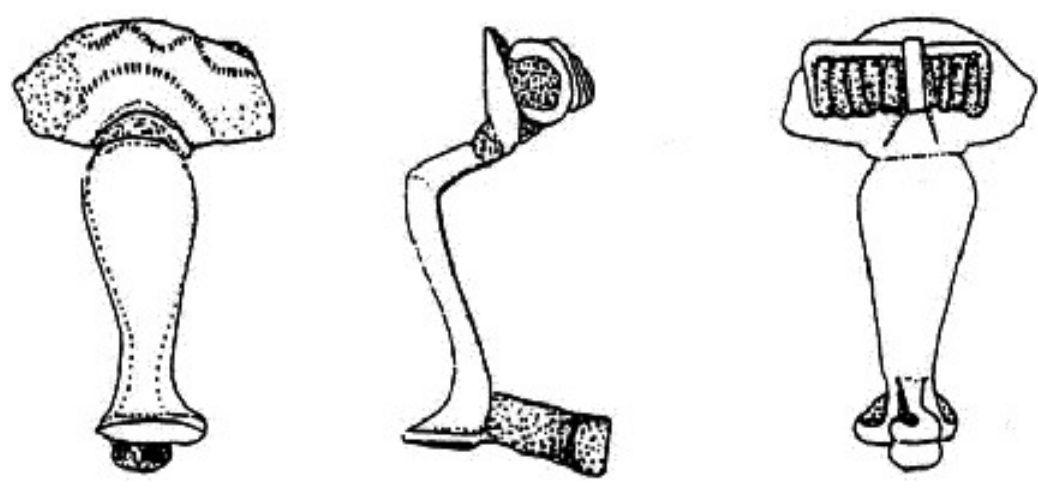

1
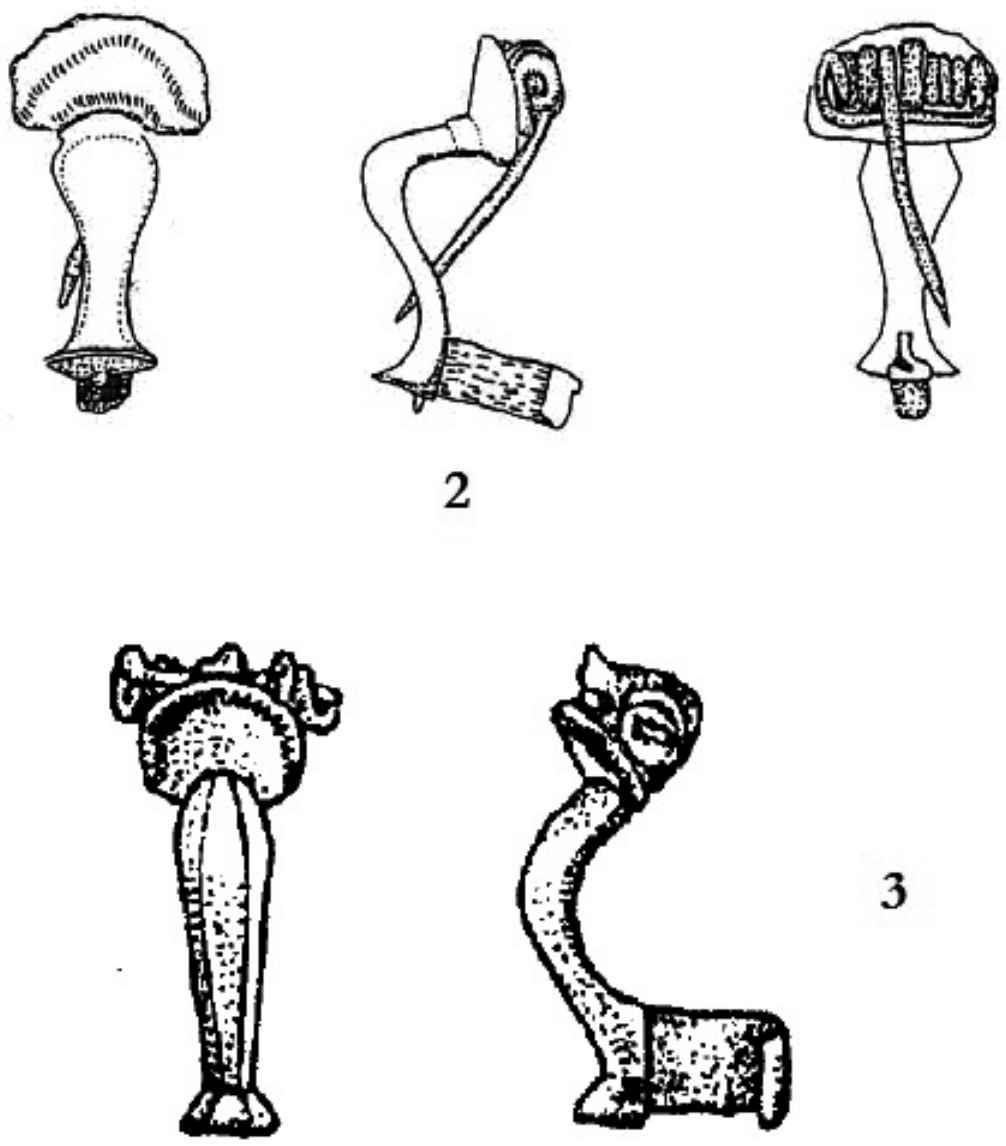

3

FIG. 3. Fíbulas de rodilla (Kniefibeln) procedentes de: 1 y 2. Flavia Solva, Austria (Kropf y Nowak, 1998-1999: lám 18, n. ${ }^{\circ} 93$ y lám. 20, n. ${ }^{\circ}$ 101); 3. Campamento de Gilau, Rumanía (Isac y Cocis, 1995: 133, n. 31$)$. 


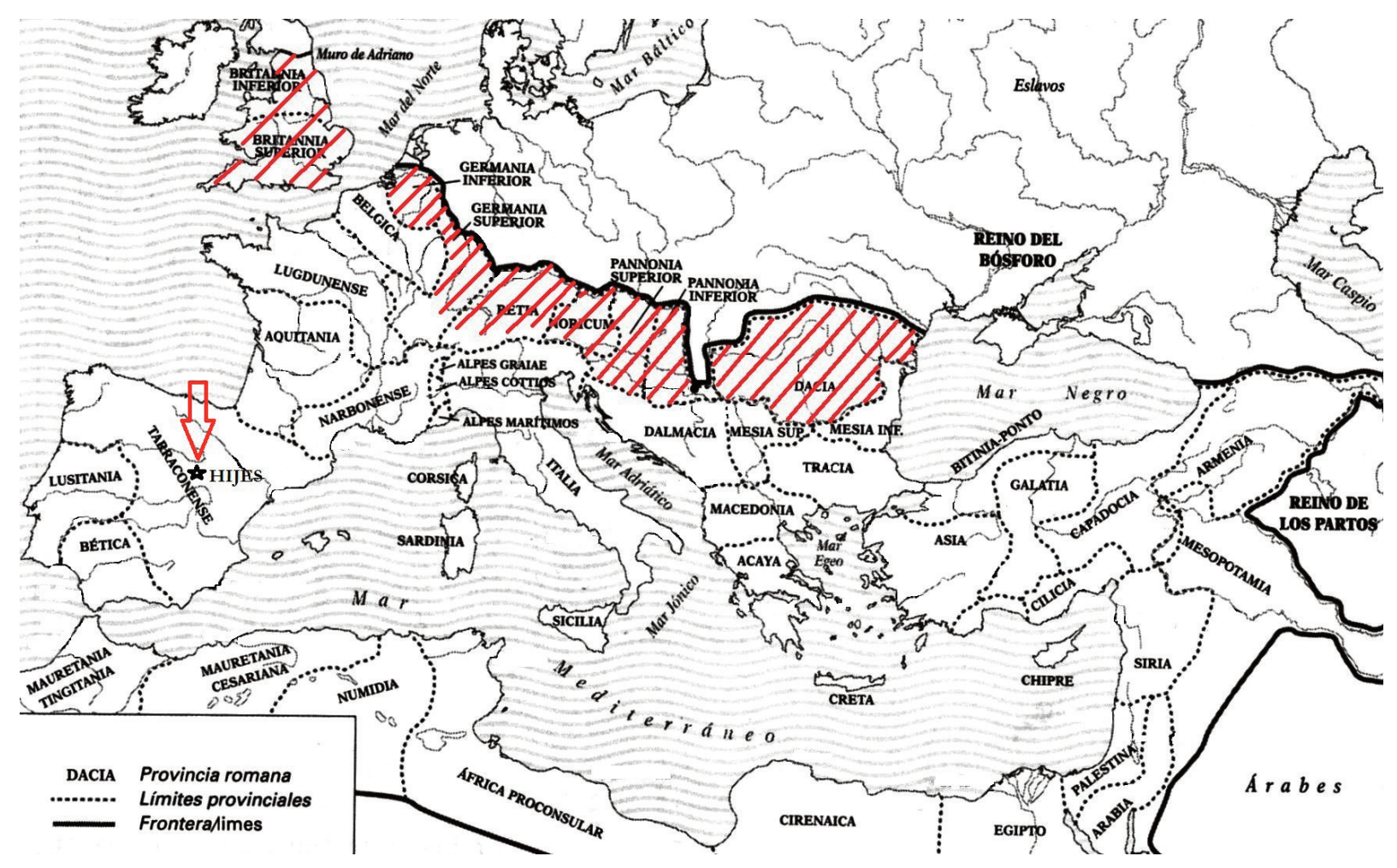

FIG. 4. Distribución geográfica de las fibulas de rodilla.

lugar de origen, trajeron consigo algunas de las pertenencias adquiridas en campańa. Pero aparte de esta afirmación genérica, podemos aventurar que el usuario de la fíbula procedía del territorio de la ciudad de Tiermes.

La atribución de su propietario al territorio termestino parece avalada por dos indicios. El primero de ellos es que la fíbula procede del enclave romano de Hijes, a $17 \mathrm{~km}$ de Tiermes y, por tanto, dentro de su territorio administrativo. Y el segundo es el epígrafe funerario dedicado a un individuo procedente de Tiermes 5 hallado en Worms (Renania-Palatinado), antiguo campamento romano de Borbetomagus en Germania Superior.

Dicha estela fue fechada por Boopert (1998: 56, lám. 58) en época tiberiana y en ella se constata

5 El texto de la estela funeraria CIL XIII, 6236 es: M(arcus) SEMPRONI/VS L(uci) F(ilius) DOMO/ TERMESTINVS/ ANNO(rum) XX[...]/ DEC(urio) EQUES ALAE/ SEBOSIANAE/ $H$ (ic) $S$ (itus) $E(s t)$. que este individuo era un decurión procedente de Tiermes que perteneció al ala Augusta Gallorum Sebosiana, hecho interesante porque fue un cuerpo de caballería auxiliar que estuvo en Hispania en época julio-claudia (García y Bellido, 1961: 134; Spaul, 1994; Carretero, 1999: 145, 149; Santos, 2007: 81). Esto indica que desde muy pronto fue habitual que gentes de la Tarraconense se enrolaran en las tropas que participaron en la defensa del limes germánico. La presencia de este cuerpo auxiliar en Hispania se ha datado a partir de la inscripción de una lápida encontrada en Italia Central dedicada a un personaje que fue prefecto de esa milicia y también tribuno militar de la legio X Gemina, que dispuso del ala II Gallorum como unidad auxiliar y que salió de Hispania hacia el año $62 \mathrm{~d}$. C. (García y Bellido, 1961: 134; Santos, 2007: 81).

Se sabe que hubo muchos emigrantes que salieron del territorio de Tiermes en los primeros siglos del imperio romano, pues se han documentado epígrafes alusivos a termestinos en varias regiones peninsulares: en Ávila (Hernando, 2005: 
87-90, n.o 12-13), Almonaster la Real (Huelva), Salamanca (Jimeno, 1980: 253), Uxama (Jimeno, 1980: 155, n.o 132) y Mérida (HEp 7, 127; EE VIII, 346, n.o 23). Aunque muchos de los movimientos de personas documentados a partir de la epigrafía se han relacionado con la ganadería trashumante (Gómez-Pantoja, 1999: 106), no hay que descartar que también se debieron a otro tipo de actividades como su enrolamiento en el ejército, siempre móvil. La presencia de termestinos en lugares tan alejados de Europa avala esta última hipótesis y está claro que, al igual que otros militares de diferentes provincias del imperio, formaron parte de unidades enviadas para la defensa del limes germánico en el s. II d. C.

Los movimientos de contingentes militares durante el imperio romano entre distintas provincias del imperio, incluida Hispania (Aurrecoechea, 1996; Morillo, 2007), han sido analizados no sólo desde el punto de vista político y militar, sino también desde el estrictamente arqueológico puesto que explican la difusión de determinados elementos de cultura material.

\section{Consideraciones finales}

Consideramos obligado prestar atención a esta fíbula de rodilla ya que es demostrativa de que el estudio de objetos significativos, con tipología y cronología bien establecidas y además hallados en contextos poco habituales, puede conducir a la identificación de usos específicos de ciertas piezas así como de las relaciones de intercambio y desplazamientos humanos. La valoración tipológica de un objeto alcanza mayor precisión cuando se puede relacionar con las informaciones procedentes de diferentes campos científicos que en este caso son la arqueología, la epigrafía y las fuentes históricas.

El análisis de los datos disponibles confirma que la novedosa presencia de este tipo de fíbula en la Meseta pudo deberse al desplazamiento de individuos oriundos del territorio de Tiermes por todo el ámbito del imperio que participaron activamente en las instituciones militares. Así pues, constituye un nuevo testimonio de la movilidad del ejército romano que mediante el desplazamiento de gentes de distinta procedencia favoreció la difusión de objetos entre unos territorios y otros.

La presencia de este modelo de fíbula en Hijes y su significado confirma también el interés que puede tener la revisión de viejas colecciones de materiales arqueológicos, siempre que se apliquen nuevas perspectivas para su interpretación y vaya precedida de un sistemático trabajo de campo que permita comprobar la veracidad de las informaciones conservadas.

\section{Bibliografía}

Aguilera y GAmbOA, E. (1916): Las necrópolis ibéricas. Madrid.

AMARÉ, M. T. (1987): "Lucernas romanas del extremo sur del convento cesaraugustano: provincias de Guadalajara y Madrid", Wad-Al-Hayara, 14, pp. 9-34.

Aurrecoechea, J. (1996): "Nuevas aportaciones al conocimiento de los contingentes militares tardorromanos en Hispania: la guarnición de cinturón de origen británico encontrado en Iruña", Veleia, 13, pp. 265-270.

Berecz, K. (1991): "Római kori fibulák Zala megyéböl”, Zalai Muzeum, 3, pp. 163-183.

BogDAN, D. y Cocis, S. (2006): "Roman brooches from Apulum", Apulum: Archeologie. Istorie. Etnografie, 43-1, pp. 219-230.

BOOPERT, W. (1998): Romische Steindenkmdler aus Worms und Umgebung. Mainz.

CARretero, S. (1999): "El ejército romano del noroeste peninsular durante el Alto Imperio", Gladius, XIX, pp. 143-156. http://dx.doi.org/10.3989/gladius.1999.17

Castro, P.; Escoriza, T. y Sanahuja, E. (2004): “A la búsqueda de las mujeres y los hombres. Sujetos sociales, espacios estructurados y análisis de materiales en un proyecto de arqueología prehistórica”. En Avances en Arqueometría 2003. Cádiz: Univ. Cádiz, pp. 251-259.

ERICE, R. (1995): Las fibulas del nordeste de la Peninsula Ibérica: siglos I a. e. al IV d. e. Zaragoza: Institución Fernando el Católico.

García y Bellido, A. (1961): "El exercitus hispanicus desde Augusto a Vespasiano", Archivo Español de Arqueología, 34, n. ${ }^{\circ}$ 103-104, pp. 114-160.

GÓmez-PANTOJA, J. (1999): "Historia de dos ciudades: Capera y Clunia". En Rodríguez MarTín, F. G. y GORGES, J.-G. (coords.): Économie et territoire en Lusitanie romaine. Madrid, pp. 91-108.

Gómez-Pantoja, J. y López, M. A. (2004): "Los inicios de la arqueología en Guadalajara. Dos notas sobre yacimientos poco conocidos". En GómEZ- 
Pantoja, J. (ed.): Excavando papeles. Indagaciones arqueológicas en los archivos españoles. Guadalajara: ed. Aache, pp. 153-166.

HatтATt, R. (1985): Iron Age and Roman Brooches. A Second Selection of Brooches from the Author's Collection. Oxford.

Hernando, R. (2005): Epigrafía romana de Ávila. Petrae Hispaniarum, 3. Bordeaux: AUSONIUS éditions.

ISAC, D. y CoCIS, S. (1995): "Fibule din castrele romane de la Gilau si Casieu. O analiza in context stratigrafic", Ephemeris Napocensis, v, pp. 103-138.

JimENO, A. (1980): Epigrafía romana de la provincia de Soria. Soria: Diput. Prov. de Soria.

JoBST, W. (1975): Die romischen Fibeln aus Lauriacum. Forschungen in Lauriacum, 10. Linz.

KovrIG, I. (1937): Die Haupttypen der kaiserzeitlichen Fibeln in Pannonien. Dissertationes Pannonicae, Series II, 4. Budapest.

KROPF, J. y NOWAK, H. (1998-1999): Fibeln von Flavia Solva aus privatbesitz. Römisches Österreich, 21-22. Viena.
MARINÉ, M. (2001): Las fibulas romanas en Hispania: La Meseta. Madrid: CSIC.

- (2007): "Las fíbulas en la España romana: alfileres para la historia", Sautuola, 13, pp. 131-144.

Morillo, A. (coord.) (2007): El ejército romano en Hispania: guía arqueológica, León: Universidad de León.

PATEK, E. (1942): Verbreitung und Herkunft der romischen Fibeltypen von Pannonien. Dissertationes Pannonicae, Series II, 19. Budapest.

Ponte, S. (2007): "Las fíbulas romanas de Portugal", Sautuola, 13, pp. 145-166.

RiHA, E. (1979): Die romischen Fibeln aus Augst und Kaiseraugst. Forschungen in Augst, 3. Augst.

Santos, N. (2007): "El ejército romano de conquista en el norte de la Península Ibérica", Historia Antiqua, 31, pp. 51-86.

SpaUl, J. (1994): Ala: The auxiliary calvary units of the pre-Diocletianic Imperial Roman Army. Andover.

VADAY, A. H. (1989): "Die sarmatischen Denkmäler des Komitats Szolnok", Antaeus, 17-18, pp. 9-353. 